\title{
Human Papilloma Virus Vaccine for the Prevention of Cancer Cervix - Awareness and Acceptability: A Study in Urban Population of UT, Chandigarh (Hospital Based): A Prospective Observational Study
}

\author{
Aashima Arora ${ }^{1}$, Vanita Suri ${ }^{2}$, Neelam Aggarwal ${ }^{3}$, Anju Singh ${ }^{4}$
}

\begin{abstract}
Cervical cancer is the most common cancer in Indian females. India reports one-fourth of the total world's cervical cancer each year that results in $17 \%$ of all women's death from this disease globally. More than $80 \%$ of cervical cancer in India is caused by human papilloma virus (HPV). The current study aimed to assess the level of awareness and acceptability of HPV vaccine among urban women attending outpatient department (OPD) of Gynecology, PGIMER, Chandigarh.

Materials and methods: A total of 200 women attending the OPD of Gynecology, PGIMER, Chandigarh, for any gynecological complaint were included in the study. Inclusion criteria included sexually active adult females with willingness to participate in the survey. All participants were assessed for the etiology of cancer cervix and available screening modalities for cervical precancerous lesions using a preformed questionnaire. Special emphasis was laid on - whether the subjects were aware about its availability, cost, dosing schedule, and the efficacy of HPV vaccine. Result: Among all, $11 \%$ of subjects were aware that early coitus and early pregnancy are predisposing factor for cervical cancer. Human papilloma virus is sexually transmitted infection and the most common cause of cervical cancer was known to $13 \%$ of subjects; however, $<3 \%$ were aware that HPV infection is transient and there is no treatment available for this. About $25 \%$ subjects were aware that Pap smear was the screening modality. Only $11 \%$ were aware that cervical cancer can be prevented by HPV vaccine.

Conclusion: There is a lack of awareness for the etiology, predisposing factors, available screening method, and vaccine for the prevention of cervical cancers among sexually active Indian females. Further emphasis on the awareness of HPV infection and vaccine should be made among female population.
\end{abstract}

Keywords: Carcinoma, Cervix, Human papilloma virus vaccine.

Journal of Postgraduate Medicine, Education and Research (2021): 10.5005/jp-journals-10028-1371

\section{INTRODUCTION}

Cervical cancer is the most common cancer in Indian females and represents the second most common cancer among females worldwide. India reports one-fourth of the total world's cervical cancer cases each year that represent $17 \%$ of all women's death from this disease globally between 30 years and 60 years. ${ }^{1,2}$ It is estimated that cervical cancer will occur in approximately 1 in 53 Indian women during their lifetime compared with 1 in 100 women in more developed regions of the world. According to the national cancer registry, cervical cancer accounts for $40 \%$ of all cancers in urban population and $65 \%$ of all cancers in the rural population. ${ }^{3}$ Despite measures available to detect this disease in precancerous stage, its incidence remain high in this part of the world, probably because of the lack of awareness.

Human papilloma virus is the cause for $>80 \%$ of cervical cancers in India. ${ }^{4}$ It is also the most common sexually transmitted infections worldwide. About $50-80 \%$ of all sexually active individuals may contract HPV at some point in their lifetime. The infection persists in $8-10 \%$ of the subjects and causes cancer in $<1 \%$ of these. Widespread knowledge about HPV infection, its sequelae, and routine screening for cervical cancerous and precancerous lesions have resulted in low incidence of cervical cancer in the developed world. At present, cervical cancer has been ranked down as the eighth most common cancer in US. ${ }^{5}$ More than $80 \%$ of all new cases
${ }^{1-4}$ Department of Obstetrics and Gynecology, Postgraduate Institute of Medical Education and Research, Chandigarh, India

Corresponding Author: Anju Singh, Department of Obstetrics and Gynecology, Postgraduate Institute of Medical Education and Research, Chandigarh, India, Phone: +91 9646121162, e-mail: singha1712@gmail.com

How to cite this article: Arora A, Suri V, Aggarwal N, et al. Human Papilloma Virus Vaccine for the Prevention of Cancer CervixAwareness and Acceptability: A Study in Urban Population of UT, Chandigarh (Hospital Based): A Prospective Observational Study. J Postgrad Med Edu Res 2021;55(2):70-73.

Source of support: Nil

Conflict of interest: None

are being reported from the developing countries. Hence, the need of the hour is to spread awareness among masses regarding the disease's etiology, screening, and preventive measures.

In the West, there have been studies to assess the knowledge of HPV infection and acceptability of its vaccine. ${ }^{6-8}$ However, to the best of our knowledge, there are no such study that have focused on this issue in the developing countries. The aim of the current study is to investigate the level of awareness regarding cervical cancer etiology, available screening methods, and the HPV vaccine among women. 


\section{Materials and Methods}

After obtaining waiver from institute ethic committee, a total of 200 women, attending the OPD of Gynecology, PGIMER, Chandigarh for any gynecological complaint were selected for this study. Inclusion criteria included females aged 18-70 years, sexually active, and willingness to participate in the brief survey. All potential participants were approached by a bilingual research officer in the waiting area of the OPD and invited to participate. Those who agreed were asked a set of questionnaire in a separate room and were given the option of answering in English/Hindi.

The demographic and socioeconomic profile of participants was recorded including obstetric details and history of smoking and alcohol/drug intake in both subject and their male partner. The questions about etiology of cancer cervix and HPV were in the format of yes/no. Awareness regarding screening modalities of cervical precancerous lesions was also assessed. Special emphasis was laid on knowledge about HPV vaccine-whether the subjects were aware about its availability, cost, dosing schedule, and efficacy. The acceptability of the vaccine for self as well as for unmarried daughters was also asked about. To assess whether cost was a hurdle in widespread use of vaccine, it was asked directly whether subjects would like vaccine to be provided free by the government. The answers to the questionnaire were recorded in a preformed format and thereafter these women were provided detailed knowledge about the subject by the research officer.

\section{Observations and Results}

A total of 200 non-pregnant women from 21 to 67 years of age participated in this survey. Of these, 139 were residing in urban areas while 61 hailed from villages. The education status, occupation, income, and religion of the women and partners are depicted in Table 1.

Out of the 200 women, 99 were primiparous while 101 were multiparous. One hundred and fifty-four subjects had one daughter each while 36 had two or more daughters. None of the women were smoker or alcoholic. Male partners of 6 women were chronic smokers and 14 partners were alcoholic.

\section{Knowledge about Cancer Etiology}

Only $11 \%$ of the women were aware that early age at first coitus and early age at first pregnancy were predisposing factors for cervical cancer. Only $19 \%$ women knew that multiple sexual partners and multiple partners of husband had a role in etiology of cervical cancer. Only $4 \%$ of the subjects knew about the role of smoking of self and partner in causation of the disease.

Out of six predisposing factors of cervical cancer (i.e., early age at first coitus and at first pregnancy, multiple sexual partners of self/ partner, smoking by self/partner), 157 women were not aware of even about a single predisposing factor for the disease (Fig. 1). Only $12 \%$ of the women knew about three or more of these predisposing factors (Fig. 1).

\section{Knowledge about HPV Infection}

The knowledge about HPV infection was assessed in the form of five questions. Only $13 \%$ of the subjects knew that HPV is a sexually transmitted infection and is the most common cause of cervical cancer. Less than $3 \%$ were aware that it is a transient infection and no treatment is available for the same. Hardly $10 \%$ of the women
Table 1: The education status, occupation, income, and religion of the subjects and their partners

\begin{tabular}{lcc}
\hline & Number of subjects & Percentage \\
\hline Education status & & \\
Illiterate & 19 & 9.5 \\
Up to high school & 51 & 25.5 \\
Senior secondary & 45 & 22.5 \\
Graduate or more & 85 & 42.5 \\
Occupation: wife & & \\
Housewife & 139 & 69.5 \\
Self-employed & 30 & 15 \\
Govt employee & 31 & 15.5 \\
Occupation: husband & & \\
Labor work & 18 & 9 \\
Self-employed & 136 & 68 \\
Govt employed & 46 & 23 \\
Monthly income & & \\
$<5,000$ & 17 & 8.5 \\
5,000-20,000 & 112 & 56 \\
$>$ 20,000 & 71 & 35.5 \\
Religion & & 27 \\
Hindu & 137 & 2.5 \\
Muslim & 4 & \\
Sikh & 54 & \\
Christian & 5 & \\
\hline & & \\
\hline & &
\end{tabular}

were aware that the incidence of HPV infection increased with exposure to multiple sexual partners (Fig. 2).

\section{Knowledge about Cervical Cancer Screening and Prevention}

Around one-fourth of the subjects (53) were aware that cancer cervix can be prevented by regular screening. All of these subjects knew Pap smear as the screening modality. However, knowledge about when to start Pap smear and when to repeat was known to only $15 \%$. Human papilloma virus DNA, as screening, was known to around $9 \%$ of the subjects while visual inspection with acetic acid (VIA) was known to only $4 \%$.

\section{HPV Vaccine Awareness and Acceptability}

Only $11 \%$ of the women were aware that cervical cancer can be prevented by vaccine. Those who were aware of the vaccine also knew that young girls (9-26 years) should be vaccinated before first sexual exposure; however, details about dosing schedule was not known to any. Even after providing information about the vaccine, $30 \%$ of the women refused to accept it for their unmarried daughters. They stated various reasons for this unacceptability; elder family members not approving of it and effect on future fertility being the most common concerns. The cost of the vaccine was another important concern; most of the women felt that the vaccine should be provided free of cost by the government of India.

\section{Discussion}

Human papilloma virus is the most common sexually transmitted infection, causing a substantial burden of disease in both men and women worldwide. Human papilloma virus causes $>75 \%$ of cervical cancers. More than half a million new cases of this cancer 


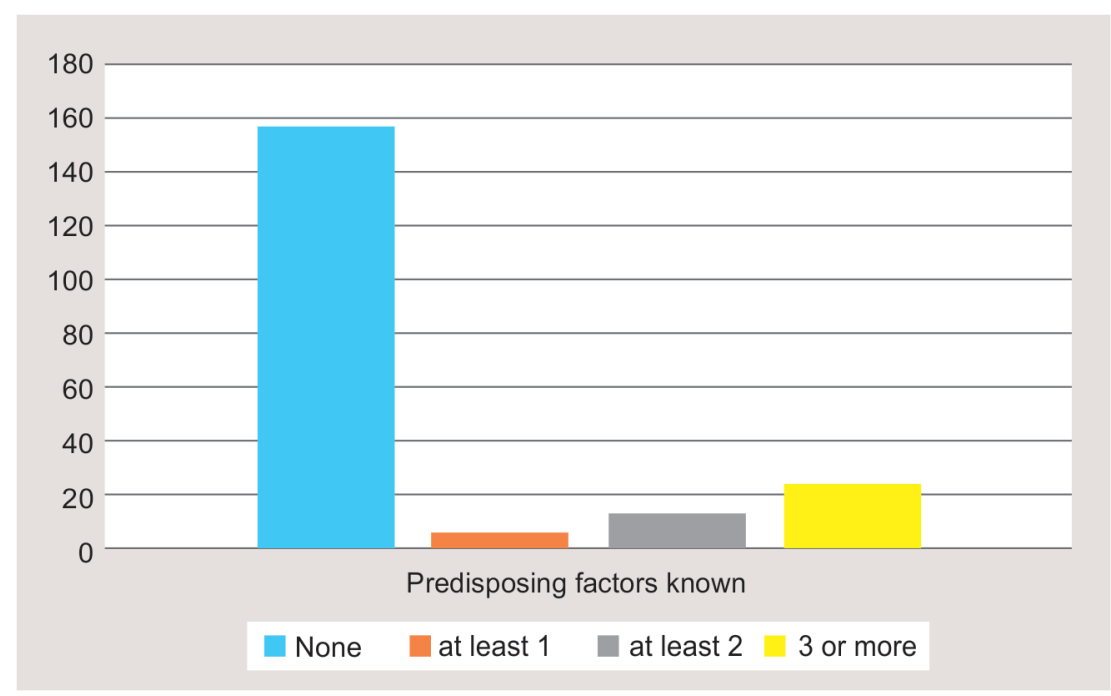

Fig. 1: Knowledge of predisposing factors among studied subjects

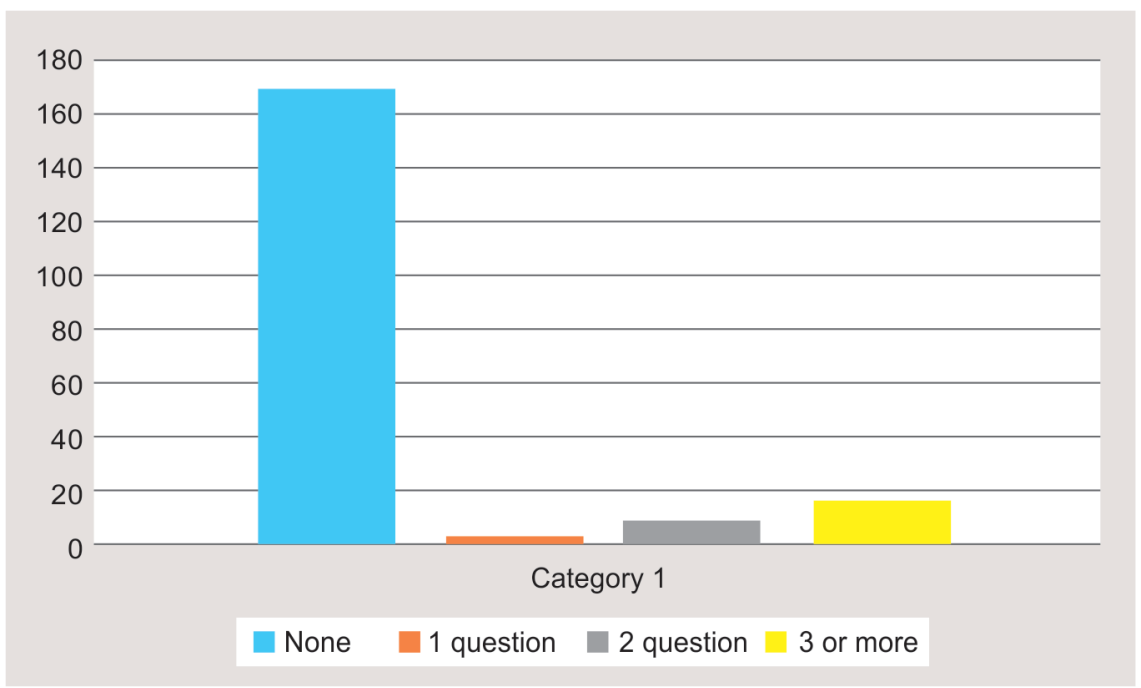

Fig. 2: Knowledge about human papilloma virus

are reported annually worldwide; $80 \%$ occurring in the developing countries. The disease kills around 3 lakh women in the prime of their lives each year; one-fourth being from India. The two HPV vaccines licensed globally are also available in India; a quadrivalent vaccine (Gardasil ${ }^{\mathrm{TM}}$ marketed by Merck) and a bivalent vaccine (Cervarix ${ }^{\mathrm{TM}}$ marketed by Glaxo Smith Kline). ${ }^{9}$ The prophylactic HPV vaccine is safe, effective, and has the potential to substantially reduce morbidity and mortality; therefore, universal vaccination is now encouraged by public health professionals. However, despite recommendation for its routine use, vaccination coverage rates remain as low as $<10 \%$ in the developing countries.

An understanding of demographic, sociocultural, and behavioral factors associated with vaccine uptake for all individuals is a priority. Previous research has focused on HPV vaccination among White women. The widespread awareness about cervical cancer screening modalities and routine vaccination have brought down the incidence of this disease in the West. At present, a marked disparity exists in the incidence and prevalence of HPV-associated cervical cancer between the White and Blacks. The purpose of this study was to understand the extent of awareness regarding cervical cancer etiology and prevention among general population in a developing country. Special emphasis was laid on assessing factors associated with HPV vaccine knowledge and acceptability. The study illustrates a shocking state of lack of awareness about this subject among women of reproductive age group in India.

The present study found that the factors predisposing to cervical cancer were known to $<20 \%$ of the population. Hence, in contrast to the developed countries where the focus is on HPV vaccine acceptability, India stands at a state where masses are not even aware of the factors that predispose them to this disease. In a previous study by Divakar, $19.7 \%$ of school students and $26.2 \%$ of urban women were aware of multiple sexual partners as risk factor of cervical cancer. ${ }^{10}$ They found that more than one-third of urban women as well as school children were aware that cervical cancer is caused by an infection; however, the details about HPV and its predisposing factors were not known to many. On the other hand, our study depicted that HPV as the cause of cervical cancer was known to only $13 \%$ of the general population. The lower level of awareness in our study could have been because of the cohort being a mixed population with $35 \%$ educated upto high school 
or less and about $30 \%$ hailing from rural areas. Shah et al. in their cross-sectional interview-based survey among 100 staff nurses found that only $11.5 \%$ respondents were aware of multiple sexual partners as one of the risk factors for cervical carcinoma; though other factors, such as early marriage and early pregnancy, were better known among them ( $71 \%$ and $74 \%$, respectively). ${ }^{11}$ A similar survey conducted in the nursing staff of tertiary care hospitals of Pakistan by Ali et al. showed that $45 \%$ mentioned multiple partners and other promiscuous behavior as the most common risk factor. ${ }^{12}$ However, most of these studies have not focused specifically on $\mathrm{HPV}$ infection as the cause of cervical cancer.

Only 22 of the 200 women knew that cervical cancer can be prevented by vaccination. Those who were aware of the vaccine also knew that young girls (9-26 years) should be vaccinated before sexual exposure; however, details about dosing schedule was not known to any. Even when told about the vaccine, 30\% of the women refused to accept it for their unmarried daughters. They stated various causes for this unacceptability; risk of any side effects and any possible effect on future fertility being the most common concerns. This concern has also been seen in previous studies on acceptability of HPV vaccine. Also, some conservative parents felt that they did not want to discuss HPV vaccination with their children because of the concern that vaccination may promote promiscuous sexual behavior.

The cost of the vaccine was another important concern for most women; three-fourth of the total women felt that the vaccine should be provided free of cost by the government. Cervical cancer kills around 3 lakh women in the prime of their lives each year; one-fourth of these are from India. Therefore, in view of this high prevalence of the disease in the country and the potential of the vaccine to substantially reduce the morbidity and mortality because of this disease; the inclusion of the vaccine in the national program must be considered.

\section{Conclusion}

There is lack of awareness for the etiology, predisposing factors, available screening method for early detection, and vaccine for the prevention of cervical cancers among sexually active Indian females. In view of this high prevalence of the disease in the country and the potential of the vaccine to substantially reduce the morbidity and mortality; awareness of HPV infection and vaccine should be made among female population.

\section{References}

1. Ferlay R, Soerjomataram R, Ervik R, et al. GLOBOCAN 2012, cancer incidence and mortality worldwide in 2012. Int J Cancer 2015;136(5):E359-E386. DOI: 10.1002/ijc.29210. Available from: http:// www. globocan.iarc.fr. [Last accessed on 2015 Dec 03].

2. Institute for Health Metrics and Evaluation. The Challenge Ahead: Progress in Breast and Cervical Cancer. Institute of Health Metrics and Evaluation; 2011. Available from: http://www. healthmetricsandevaluation.org/publications/policyreport/ challenge-ahead-progress-and-setbacks-breastand-cervical-cancer. [Last accessed on 2016 Jan 21].

3. Nandakumar A, Ramnath T, Chaturvedi M. The magnitude of cancer cervix in India. Indian J Med Res 2009;130(3):219-221.

4. Bharadwaj M, Hussain S, Nasare V, et al. HPV \& HPV vaccination: issues in developing countries. Indian J Med Res 2009;130(3):327-333.

5. Adegoke $O$, Kulasingam S, Virnig B. Cervical cancer trends in the United States: A 35-year population-based analysis. J Womens Health (Larchmt) 2012;21(10):1031-1037. DOI: 10.1089/jwh.2011.3385.

6. Holman DM, Benard V, Roland KB, et al. Barriers to human papillomavirus vaccination among US adolescents: A systematic review of the literature. JAMA Pediatr 2014;168:76-82. DOI: 10.1001/ jamapediatrics.2013.2752.

7. Waller J, Ostini R, Marlow LA, et al. Validation of a measure of knowledge about human papillomavirus (HPV) using item response theory and classical test theory. Prev Med 2013;56(1):35-40. DOI: 10.1016/j.ypmed.2012.10.028.

8. Fowler B, Bodson J, Warner EL, et al. Poor HPV vaccine-related awareness and knowledge among Utah latinas overdue for recommended cancer screenings. J Community Health 2016;41: 825-837. DOI: 10.1007/s10900-016-0160-3.

9. Singhal T. Indian academy of pediatrics committee on immunisation (IAPCOI) - consensus recommendations on immunization. Indian Pediatr 2008;45:635-648.

10. Divakar H. Knowledge and awareness about preventive health seeking behavior and acceptability of cervical cancer vaccine in urban women in comparison with school students. J South Asian Feder Obst Gynae 2012;4(1):47-53. DOI: 10.5005/jp-journals10006-1172.

11. Shah V, Vyas S, Singh A, et al. Awareness and knowledge of cervical cancer and its prevention among the nursing staff of a tertiary health institute in Ahmedabad, Gujarat, India. Ecancermedicalscience 2012;6:270.

12. Ali SF, Ayub S, Manzoor NF, et al. Knowledge and awareness about cervical cancer and its prevention amongst interns and nursing staff in tertiary care hospitals in Karachi, Pakistan. PLoS One 2010;5(6):e11059. DOI: 10.1371/journal.pone.0011059. 